\title{
Number Fluctuation in an interacting trapped gas in one and two dimensions
}

\author{
R. K. Bhaduri ${ }^{1}$, M. V. N. Murthy and Muoi N. Tran ${ }^{1}$ \\ The Institute of Mathematical Sciences \\ Chennai 600 113, India \\ 1. Permanent Address: Department of Physics and Astronomy, \\ McMaster University,Hamilton, Ontario, \\ Canada L8S $4 M 1$
}

\begin{abstract}
It is well-known that the number fluctuation in the grand canonical ensemble, which is directly proportional to the compressibility, diverges for an ideal bose gas as $T \rightarrow 0$. We show that this divergence is removed when the atoms interact in one dimension through an inverse square two-body interaction. In two dimensions, similar results are obtained using a self-consistent ThomasFermi (TF) model for a repulsive zero-range interaction. Both models may be mapped on to a system of non-interacting particles obeying the Haldane$\mathrm{Wu}$ exclusion statistics. We also calculate the number fluctuation from the ground state of the gas in these interacting models, and compare the grand canonical results with those obtained from the canonical ensemble.
\end{abstract}




\section{INTRODUCTION}

Consider an ideal bose gas at low temperatures. In the grand canonical ensemble (GCE), the compressibility and the number fluctuation of this system diverge at low temperatures [1]. To quote Landau and Lifshitz [1]:

...in a bose gas at temperature $T<T_{c}$, the pressure is independent of the volume, i.e. the compressibility becomes infinite. Accordingly...this would imply that the fluctuations of the number of particles also become infinite. This means that, in calculating fluctuations in a gas obeying bose statistics, the interactions between the particles cannot be neglected at low temperatures, however weak this interaction may be. When the interactions, which must exist in any actual gas, is taken into account, the resulting fluctuations are finite.

One purpose of this paper is to demonstrate the validity of this statement in one and two dimensional traps for a special class of interactions.

In one dimension, the quantum many-body problem of particles in a harmonic oscillator interacting with an inverse square two-body potential is exactly solvable [2,3]. Moreover, it is known that the global properties of these interacting bosons are the same as those of non-interacting particles obeying the Haldane-Wu generalized exclusion statistics (ideal haldons) [ [6,5]. Using this mapping, we show that the number fluctuation is finite as $T \rightarrow 0$, no matter how weak the interaction strength is. In this model, since the exact correlation function is known for some specific strength parameters of the interaction [3], it is also possible to verify explicitly the well-known relation between its integral and the number fluctuation.

In two dimensions, there is no suitable exactly solvable model for our purpose. We therefore consider the mean-field model of bosons trapped in a harmonic oscillator, and interacting pair-wise with a zero-range repulsive pseudo-potential [6]. In the absence of this interaction, the number fluctuation diverges at $T=T_{c}$. When the interaction is present, however weak, we show that the compressibility and hence the number fluctuation of the system is finite right down to $T=0$. Moreover, they are shown to be identical to those of a collection of non-interacting haldons.

Another quantity of some interest is the number fluctuation of particles from the ground state of the system, which is present even when the total number of particles in the trap is fixed. Consider a dilute gas of bosons in a trap at $T=0$. The system is in its ground state. When a certain amount of excitation energy is given to the system, it may be absorbed in many possible ways, so that the number of bosons remaining in the ground state is not

fixed. This number fluctuation for non-interacting bosons in a harmonic trap has been calculated by a number of authors [9] as a function of the excitation energy or temperature. Thermodynamic identities and particle number fluctuations in weakly interacting BEC have also been analysed when the particle number is fixed [10]. For ideal bosons, the number fluctuation from the ground state diverges at low temperatures in GCE, but this can be avoided using more careful canonical, or microcanonical treatment. When the inverse square pair-wise interaction is used in one dimension, this divergence is again removed. Further, in this interacting model, we can also perform the canonical ensemble (CE) calculations, and compare with the GCE results. We find that even though the ground state number 
fluctuation goes to zero as $T \rightarrow 0$ in both GCE and CE, the very low-temperature behaviors are rather different. At higher temperatures the results from CE and GCE tend to coincide. Similar studies can be made for fermionic systems at low temperatures. Since the interactions that we have used are of statistical character [5,11], our results interpolate from bosonic to fermionic behavior with the variation in the strength of the interaction.

The outline of the paper is as follows: Sect. II deals with the total number fluctuation in GCE for interacting models. Sect. II (a) is devoted to a discussion of the fluctuations in one dimension, and II (b) to fluctuation in two dimensions. In Sect. III, the ground state number fluctuations for the one-dimensional interacting model in GCE and CE are calculated and compared. Particular attention is paid to the low temperature behavior (see also Appendix). We conclude the paper with a brief discussion of the results.

\section{FLUCTUATIONS IN GCE}

The number fluctuation in a gas in the GCE formalism is defined by $(\delta N)^{2}=\left(<N^{2}>\right.$ $-<N>^{2}$ ), where the angular brackets denote ensemble averaging. For an ideal bose gas,

$$
(\delta N)^{2}=\sum_{k=0}^{\infty}\left\langle n_{k}\right\rangle\left(\left\langle n_{k}\right\rangle+1\right),
$$

where the single particle occupancy $\left\langle n_{k}\right\rangle$ at a given temperature $T$ and energy $\epsilon_{k}$ are given by the bose distribution function

$$
\left\langle n_{k}\right\rangle=\frac{1}{\exp \left[\beta\left(\epsilon_{k}-\mu\right)\right]-1} .
$$

Here $\beta=1 / T$ with the Boltzmann constant $k_{B}=1$, and $\mu$ denotes the chemical potential. The isothermal compressibility $\chi_{T}$ of a gas of density $\rho_{0}$ is related to the number fluctuation of the system in GCE :

$$
\frac{(\delta N)^{2}}{N}=T \rho_{0} \chi_{T}
$$

The problem with the number fluctuation in a bose gas in GCE is obvious from Eq. (11). If there is BEC, then a macroscopic fraction of the particles occupy the ground state for $T<T_{c}$, so that $\left(\delta N_{0}\right)^{2} \simeq N^{2}$. Even if there is no BEC, the same is the case as $T \rightarrow 0$. In the thermodynamic limit, therefore, the fluctuation diverges below the critical temperature, or in any case at $T=0$. This is manifestly incorrect, since at $T=0$, all the bosons are in the ground state, and the number fluctuation should vanish. In the presence of interactions, however, this number fluctuation is expected to be finite. We demonstrate this by analyzing two models in one and two dimensions.

\section{A. Fluctuations in a one dimensional model}

We first consider the exactly solvable one dimensional Calogero-Sutherland Model (CSM) of a system of interacting particles with the Hamiltonian [2,3] 


$$
H=\sum_{i=1}^{N}\left[-\frac{\hbar^{2}}{2 m} \frac{\partial^{2}}{\partial x_{i}^{2}}+\frac{1}{2} m \omega^{2} x_{i}^{2}\right]+\frac{\hbar^{2}}{m} \sum_{i<j=1}^{N} \frac{g(g-1)}{\left(x_{i}-x_{j}\right)^{2}},
$$

with the dimensionless coupling parameter $g \geq 0$. The particles are confined in a harmonic well and the thermodynamic limit is obtained by taking $\omega \rightarrow 0$ as $N \rightarrow \infty$, with $\omega N=$ constant. In the thermodynamic limit, the properties of the system are translationally invariant, and would be the same if the particles were on a line, or a circle, instead of a harmonic confinement. To make the problem well-defined quantum mechanically, we have to demand that the wave functions go to zero as $\left|x_{i}-x_{j}\right|^{g}$ whenever two particles $\mathrm{i}$ and $\mathrm{j}$ approach each other. Since the particles cannot cross each other, we may choose the wave function to be either symmetric (bosonic) or antisymmetric (fermionic). For $g=0$ and 1 , the model describes free bosons and free fermions respectively.

Using the relation between the integral of the correlation function and the number fluctuation, we now show that the number fluctuation vanishes at zero temperature in the above interacting model unlike the ideal boson result in GCE. If $\nu(r)$ denotes the two-particle ground state density-density correlation function in the ground state, with $r=\left|x_{1}-x_{2}\right|$, then the number fluctuation is related to the correlation function as [1]

$$
\frac{(\delta N)^{2}}{N}-1=-\int_{-\infty}^{\infty} \nu(x) d x
$$

Note that the ground state correlation function $\nu(r)$ is defined only for $r \geq 0$. However, in computing the above integral it is necessary to assume $\nu(r)$ to be even function, and extend the domain of integration to negative values of $r$ [12]. Unlike the one-particle off-diagonal density matrix, $\nu(r)$, by definition, is related to the diagonal element of the the two-particle density matrix, and is the same in CSM for bosons or fermions. In this section we work in the bosonic basis. The correlation functions are known exactly in the CSM for three values of $g$ independent of whether the particles are bosons are fermions and are given (in the thermodynamic limit) by [3]

$$
\begin{aligned}
& g=1: \quad \nu(r)=s(r)^{2}=\left[\frac{\sin (\pi r)}{\pi r}\right]^{2} \\
& g=1 / 2: \nu(r)=s(r)^{2}+\frac{d s}{d r} \int_{r}^{\infty} d t[s(t)] \\
& g=2: \quad \nu(r)=s(2 r)^{2}-\frac{d s(2 r)}{d r} \int_{0}^{2 r} d t[s(t)]
\end{aligned}
$$

where the Fermi momentum $k_{F}$ is set equal to $\pi$ so that the maximum central density is unity. For all three forms of $\nu(r)$ given above, explicit calculations show that

$$
\int_{-\infty}^{\infty} \nu(x) d x=\int_{-\infty}^{\infty}[s(r)]^{2} d x=1
$$

independent of the value of $g$. Substituting this result in Eq.(5) it follows that for interacting bosons in CSM the fluctuation vanishes identically at $T=0$.

While we cannot obtain the exact $\nu(r)$ in CSM for all $g$, the same may be calculated for all values of $g$ in the harmonic lattice approximation. The correlation function so obtained compares very well with the exact correlation functions for $g=1 / 2,1,2$ and is given by 13 


$$
\nu(x)=\rho_{0} \sum_{n \neq 0}\left(\frac{1}{4 \pi F(n, 0)}\right)^{1 / 2} \exp \left[-\frac{\left(x \rho_{0}-n\right)^{2}}{4 F(n, 0)}\right]-\rho_{0},
$$

where $\rho_{0}=N / L$ is the average density and

$$
\lambda F(n, 0)=\frac{1}{2 \pi^{2}} \int_{0}^{\pi} d y \frac{1-\cos (y n)}{y-y^{2} / 2 \pi}=\frac{1}{2 \pi^{2}} \int_{0}^{2 \pi} d y \frac{1-\cos (y n)}{y} .
$$

The above expression is given for completeness and its exact form is not needed for further calculations. Again integrating over the real line we get a result identical to that obtained using the exact correlation functions in CSM. Thus the fluctuation vanishes identically for all $g$ in this approximation at zero temperature. However, the result does not give any indication of the behavior of the fluctuation at finite temperature. To do this we take recourse to the mapping between the CSM and the exclusion statistics first proposed by Haldane through a generalized Pauli principle [5, 17].

A crucial property of exclusion statistical interactions is that they should cause shifts in single particle energies at all scales [14] (see next section). This property is realized by a large class of one dimensional models of interacting fermions where Fermi liquid theory breaks down [15,16]. In fact it has been shown exactly that quasiparticles with nontrivial exclusion statistics exist in a class of models that are solved by the Bethe ansatz [17]. Thus the result obtained below should be, in principle, valid in a large class of models with interactions. In particular, it is well known that the interacting particle of CSM may be regarded as ideal exclusion statistics particles or simply haldons [5]. The thermodynamic properties of an ideal gas of exclusion statistics particles have been investigated widely [18 21]. The distribution function has been computed and is given by

$$
\langle n(\epsilon)\rangle=\frac{1}{(w(\epsilon)+g)},
$$

where $w(\epsilon)$ is the solution of the equation

$$
w(\epsilon)^{g}(1+w(\epsilon))^{(1-g)}=e^{\beta(\epsilon-\mu)}
$$

At zero temperature we have,

$$
\langle n\rangle=\frac{1}{g}, \quad \text { for } \epsilon_{k} \leq \mu
$$

and zero otherwise.

Note that the distribution function reduces to the usual Fermi and Bose distribution functions for $g=1$ and $g=0$ respectively and in general $g$ is regarded as the exclusion statistics parameter. Indeed, the statistical parameter $g$ is precisely the interaction strength as given in Eq. (田) in one dimension. However, the distribution function as given above is valid in general and not necessarily restricted to one dimensional models. The following discussion is therefore used for illustration in the case of one-dimensional model but not restricted to this case alone.

The number fluctuation at a given energy $\epsilon_{k}$ is given by, 


$$
\left(\delta n_{k}\right)^{2}=T \frac{\partial\left\langle n_{k}\right\rangle}{\partial \mu} .
$$

Substituting for $\left\langle n_{k}\right\rangle$ from Eq. (12), we have for total number fluctuation [18,

$$
\begin{aligned}
(\delta N)^{2} & =\sum_{k=0}^{\infty} w_{k}\left(1+w_{k}\right)\left\langle n_{k}\right\rangle^{3} \\
& =\sum_{k=0}^{\infty}\left\langle n_{k}\right\rangle\left(1-g\left\langle n_{k}\right\rangle\right)\left(1+(1-g)\left\langle n_{k}\right\rangle\right) .
\end{aligned}
$$

The number fluctuation vanishes at $T \rightarrow 0$ since $n_{k} \rightarrow 1 / g$ below the Fermi energy and zero otherwise. This result holds no matter how weak the interaction strength is. However, at $g=0$, the bosonic limit, the number fluctuation diverges as noted earlier. In this exactly solvable model, we have thus shown that interactions do remove the fluctuation catastrophe encountered in the ideal Bose gas.

While these results have been derived in one dimension, extension to higher dimension is non-trivial since there is no suitable exactly solvable many body model. However, it has been shown that models with short range interactions in two dimension may be regarded as obeying exclusion statistics in the mean-field picture [22]. We discuss the fluctuation in these models in the next section.

\section{B. Fluctuations in a two dimensional model}

We consider a two-dimensional system of bosons interacting via a zero-range repulsive pseudo-potential. The quantum dynamics is then approximated by the following Hamiltonian

$$
H=\sum_{i=1}^{N}\left(\frac{p_{i}^{2}}{2 m}+\frac{1}{2} m \omega^{2} r_{i}^{2}\right)+\frac{2 \pi \hbar^{2}}{m} \tilde{g} \sum_{i<j}^{N} \delta\left(\mathbf{r}_{i}-\mathbf{r}_{j}\right)
$$

where the momenta and coordinates are planar vectors. The one-body potential generated by the above zero-range interaction (including exchange) is

$$
U(n(\mathbf{r}))=\frac{2 \pi \hbar^{2}}{m} g n(\mathbf{r}), \quad g=2 \tilde{g},
$$

where $n(\mathbf{r})$ is the local number density of the system. In two-dimensions, $g \geq 0$ plays the role

of the statistical parameter, with $g=0$ for non-interacting bosons. At finite temperature, for $T>T_{c}$, the Thomas-Fermi approximation yields [6, [7]

$$
n(\mathbf{r})=\int \frac{d^{2} p /(2 \pi \hbar)^{2}}{\left[\exp \left[\left(\frac{p^{2}}{2 m}+V(r)-\mu\right) \beta\right]-1\right]},
$$

where the Thomas-Fermi mean potential $V(r)$ is given by

$$
V(\mathbf{r})=V_{0}(r)+\frac{2 \pi \hbar^{2}}{m} g n(\mathbf{r}) .
$$


Here $V_{0}(r)$ is the one-body harmonic trap. Note that Eq.(19) is valid only in the absence of a condensate. It has been shown [6,7]. however, that for a nonzero positive $g$, a self-consistent solution of this equation satisfying $\int n(r) d^{2} r=N$ may be obtained right down to $\mathrm{T}=0$. This solution has a lower free energy than the one with a condensate [8], so we may take $T_{c}=0$ for $g>0$.

The momentum integration may be done analytically:

$$
n(r)=-\frac{m}{2 \pi \hbar^{2} \beta} \ln [1-\exp [-\beta(V(r)-\mu)]]
$$

The local number fluctuation (between $r$ and $r+d r$ ) in GCE is given by

$$
(\delta N)^{2}=T \frac{\partial n(r)}{\partial \mu}
$$

where the coefficient of the temperature $T$ on the rhs is related to the compressibility. Taking the derivative of the local density with respect to the chemical potential we have

$$
(\delta N)^{2}=T \frac{m}{2 \pi \hbar^{2}} \frac{1}{\exp [(V(r)-\mu) \beta]-1+g},
$$

Note that $\mu$ is a function of temperature, and is determined by the condition that $\int n(r) d^{2} r=N$, and in the thermodynamic limit it approaches the lowest energy eigenstate as the temperature goes to zero. A few remarks on the thermodynamic limit are in order: The thermodynamic limit is reached when $N \rightarrow \infty$ and $\omega \rightarrow 0$. However, in the limit of no confinement the density of states becomes a constant and there is no critical temperature below which condensation takes place. Preserving the density of states as in a two dimensional oscillator, the condensation temperature of an ideal bose gas is given by

$$
T_{c}^{(0)}=\left(6 / \pi^{2}\right)^{1 / 2} N^{1 / 2} \omega
$$

Thus the limit $N \rightarrow \infty$ and $\omega \rightarrow 0$ is obtained keeping $T_{c}^{(0)}$ constant. In an ideal bose gas $(g=0)$, no self-consistent solution of Eq. (21) can be found for a fixed $N$ below this temperature $T_{c}^{(0)}$. However, when $g>0$ no matter how small, the self-consistent solution of Eq. (21) may then be found for all $T>0$.

In this limit the fluctuation is given by,

$$
(\delta N)^{2}=T \frac{m}{2 \pi \hbar^{2}} \frac{1}{\exp \left[\left(\frac{2 \pi \hbar^{2}}{m} g n-\mu\right) \beta\right]-1+g},
$$

where $n$ is the constant density given in the thermodynamic limit. (For the one-dimensional case, this was denoted by $\rho_{0}$ earlier.)

In the absence of interaction, $g=0$, the chemical potential $\mu$ goes to zero at $T=T_{c}$ and the above expression diverges as expected. However, as in the one dimensional case, the fluctuation remains finite and approaches zero as $T \rightarrow 0$ when $g$ is finite, however weak the interaction may be.

The same result may be obtained in the non-interacting exclusion statistics description in two-dimensions. The local density as a function of the radial coordinate is given by [6], 


$$
n(\mathbf{r})=\int \frac{d^{2} p /(2 \pi \hbar)^{2}}{[w+g]},
$$

where the local variable $w(p, r)$ is defined through Wu's equation within TF approximation

$$
w^{g}(1+w)^{1-g}=\exp \left[\beta\left(\frac{p^{2}}{2 m}+V_{0}(r)-\mu\right)\right]
$$

and $g$ is the exclusion statistics parameter which we will identify with the interaction strength in the mean-field picture. We have retained the trap potential $V_{0}$ as in the interacting picture. Once again the momentum integration may be done easily and we obtain,

$$
n(r)=\frac{m}{2 \pi \hbar^{2} \beta} \ln \left[\frac{1+w_{0}}{w_{0}}\right],
$$

where the local variable $w_{0}(r)$ is determined through

$$
w_{0}^{g}\left(1+w_{0}\right)^{1-g}=\exp \left[\beta\left(V_{0}(r)-\mu\right)\right] .
$$

The fluctuation is then found by using Eq. (22) and we have

$$
\begin{aligned}
\delta N^{2} & =T \frac{\partial n(r)}{\partial \mu} \\
& =T \frac{m}{2 \pi \hbar^{2}} \frac{1}{w_{0}+g}
\end{aligned}
$$

In the bosonic limit taking $g=0$ in Eq. (28), we have $w_{0}=\exp \left[\beta\left(V_{0}-\mu\right)\right]-1$. Substituting this in the above expression for the fluctuation in the thermodynamic limit it is easy to see that at $T_{c}$ there is divergence. However, for positive definite $g$ there is no divergence. Furthermore the equivalence between the non-interacting exclusion statistics picture and the mean-field description is established using the following relationship

$$
w_{0}(r)=\exp [\beta(V(r)-\mu)]-1
$$

where $V(r)$ is the self consistent mean-field potential. Substituting this in Eq.(30), and taking the thermodynamic limit yields Eq.(24). Note that this equivalence allows one to calculate fluctuations in either the mean field picture or in the non-interacting exclusion statistics picture. Indeed this holds for the computation of other global thermodynamic quantities as well.

\section{GROUND STATE FLUCTUATION IN THE CANONICAL ENSEMBLE}

The total number fluctuation defined in the previous section in the GCE is obtained by summing over all the single particle states. In the canonical ensemble however, the number of particles is fixed and therefore the fluctuation in particle number has to be defined with respect to a reference state. One way defining the fluctuation is to look at the ground state occupancy as a function of temperature, which is present even when the total number of particles in the trap is fixed. At $T=0$ all the particles are in the ground state. At a 
nonzero temperature (or excitation energy), there are many ways of exciting the particles from the ground state, leading to a fluctuation in the ground state population. This number fluctuation for non-interacting bosons in a harmonic trap has been calculated by a number of authors [9] as a function of the excitation energy or temperature. This has also been calculated for fermions in the $\mathrm{CE}$ by us in a previous publication [23]. Unlike the case of GCE for bosons, the ground state fluctuation in the CE is finite at all temperatures.

We may extend the analysis of fluctuations in CE to particles interacting via the inverse square pair-wise interaction in one dimension. It is more convenient to perform the calculation in the fermionic basis, although the formulae given here are applicable for both interacting fermions, or interacting bosons. In this section, quantities like energy and ground state number fluctuation of the interacting system with interaction strength $g$ will be denoted by a bracketed superscript, e.g. $E^{(g)},\left(\left(\delta N_{0}\right)^{2}\right)^{(g)}$. We recall that the spectrum of the CSM Hamiltonian given in Eq. (4) is exactly known. The states may be labeled by a set of fermionic occupation numbers $\left\{n_{k}\right\}, k=1, \ldots, \infty, n_{k}=0,1$. The energy $E^{(g)}$ of the system in the fermionic basis is then given by,

$$
E^{(g)}\left\{n_{k}\right\}=\sum_{k=1}^{\infty} \epsilon_{k} n_{k}-\omega(1-g) \frac{N(N-1)}{2},
$$

where $\epsilon_{k}=\left(k-\frac{1}{2} \hbar \omega\right)$ denotes the harmonic oscillator energy levels and $N=\sum_{k=1}^{\infty} n_{k}$. As can be seen from Eq. (32), the effect of the interaction is that each particle shifts the energy of every other particle by a constant $\hbar \omega(g-1)$. The energy functional can also be written as

$$
E^{(g)}\left\{n_{k}\right\}=\sum_{k=1}^{\infty} \epsilon_{k} n_{k}-\omega(1-g) \sum_{k_{1}<k_{2}=1}^{\infty} n_{k_{1}} n_{k_{2}} .
$$

The exact spectrum of the model is thus reproduced by an effective Hamiltonian of quasiparticles with constant density of states and constant Landau parameters. As mentioned before, this scale invariant energy shift is the basic reason for the occurrence of nontrivial exclusion statistics where $g$ plays the role of exclusion statistics parameter with $g=0,1$ for bosons and fermions.

The general canonical partition function in any basis is written in the occupation number representation as [5]

$$
Z_{N}^{(g)}=\sum_{\left\{n_{k}\right\}} e^{-\beta E^{(g)}\left\{n_{k}\right\}}
$$

Using the energy spectrum in CSM given in Eq. (32), the N-particle partition function in this one dimensional model is given by

$$
Z_{N}^{(g)}=e^{\tilde{\beta}(1-g) \frac{N(N-1)}{2}} Z_{N}^{F}
$$

where $\tilde{\beta}=\beta \hbar \omega$ and $Z_{N}^{F}$ is the $\mathrm{N}$ particle fermion partition function. Setting $g=0$, the bosonic partition function is obtained,

$$
Z_{N}^{B}=e^{\tilde{\beta} \frac{N(N-1)}{2}} Z_{N}^{F}
$$


Combining Eqs. (35) and (36) we may write the partition function for CSM as,

$$
Z_{N}^{(g)}=\left(Z_{N}^{F}\right)^{g}\left(Z_{N}^{B}\right)^{1-g}
$$

The canonical partition function given above is exact in CE and may be used for calculating the thermodynamic properties of the system in CSM within the canonical ensemble formalism. The moments of the occupation number are related to the partition function by

$$
\begin{aligned}
& \left\langle n_{k}\right\rangle^{(g)}=\frac{1}{Z_{N}^{(g)}} y_{k} \frac{\partial Z_{N}^{(g)}}{\partial y_{k}}, \\
& \left\langle n_{k}^{2}\right\rangle^{(g)}=\frac{1}{Z_{N}^{(g)}} y_{k} \frac{\partial}{\partial y_{k}}\left(y_{k} \frac{\partial Z_{N}^{(g)}}{\partial y_{k}}\right),
\end{aligned}
$$

where $y_{k}=\exp \left(-\beta \epsilon_{k}\right)$. Therefore, it follows that

$$
\left\langle\left(\delta n_{k}\right)^{2}\right\rangle^{(g)}=y_{k} \frac{\partial\left\langle n_{k}\right\rangle^{(g)}}{\partial y_{k}} .
$$

Using Eqs. (37)-(39), $\left\langle n_{k}\right\rangle(g)$ can be expressed in terms of those of fermions and bosons via:

$$
\left\langle n_{k}\right\rangle^{(g)}=g\left\langle n_{k}\right\rangle^{F}+(1-g)\left\langle n_{k}\right\rangle^{B}
$$

Unlike $\left\langle n_{k}\right\rangle^{(g)},\left\langle n_{k}^{2}\right\rangle^{(g)}$ does not have a simple form as Eq. (41). However, the expression for the fluctuation in the occupation number does:

$$
\left.\left(\left(\delta n_{k}\right)^{2}\right)^{(} g\right)=g\left(\left(\delta n_{k}\right)^{2}\right)^{F}+(1-g)\left(\left(\delta n_{k}\right)^{2}\right)^{B} .
$$

Eq. (42) gives only the fluctuation in the occupation of a given level $k$, while the quantity we are seeking is the ground state number fluctuation. The latter is formally defined in any ensemble as:

$$
\left(\delta N_{0}\right)^{2}=\sum_{k}\left(\delta n_{k}\right)^{2}=\sum_{k}\left(\left\langle n_{k}^{2}\right\rangle-\left\langle n_{k}\right\rangle^{2}\right)
$$

where the sum $k$ runs over only the levels which are completely occupied at zero temperature. Thus, in an ab initio calculation, one would formally sum over the quasiparticle levels which are occupied at $T=0$ to get $\left.\left(\left(\delta N_{0}\right)^{2}\right)^{(} g\right)$. Fig. 1 shows the level flow in CSM as a function of $g$ obtained from Eq. (33) at $T=0$. It can be seen that as $g$ changes from the fermionic to the bosonic end, the number of levels contributing to the ground state remains constant, while the Fermi energy decreases accordingly. This means that one may obtain $\left(\left(\delta N_{0}\right)^{2}\right)^{(g)}$ by simply substituting the ground state fluctuations for fermions and bosons. ie:

$$
\left(\left(\delta N_{0}\right)^{2}\right)^{(g)}=g\left(\left(\delta N_{0}\right)^{2}\right)^{F}+(1-g)\left(\left(\delta N_{0}\right)^{2}\right)^{B}
$$




\section{DISCUSSIONS}

In the previous section we have discussed the ground state number fluctuation in GCE and $\mathrm{CE}$ in an interacting system. We now compare the results obtained in these two ensembles. Due to numerical difficulty at low temperatures, we are able to do the comparison only for $N=10$ particles. Despite this limitation, some interesting points can be made. In Fig. 2(a), we show the behavior of the relative ground state fluctuation against temperature of interacting bosons for both ensembles for interacting strengths of $g=0,1 / 2,1$. The $g=0$ case corresponds to free bosons. Fig. 2(b) shows the low temperature region of the curves in Fig. 2(a). Note that as $T \rightarrow 0$ the GCE fluctuation for free bosons diverges as expected, whereas those for interacting bosons remain finite and approach zero. Note also that in the ideal Haldane gas picture the $g=1 / 2$ case corresponds to semions and the $g=1$ case is the non-interacting fermionic limit. The GCE fluctuation for semions is found using Eqs. (12), (13) and (17), where $\mu$ is determined by the constraint that the average total number of particles is $N$.

Unlike in GCE, the CE ground state fluctuation for free bosons remain finite as $T \rightarrow 0$. The ground state fluctuation of free bosons, however, approaches zero exponentially (see Fig. 2(b)), contrary to previous results that find a linear dependence with $T$ all the way to $T=0$ 925. In Appendix A we give the low temperature expansions of the fluctuation squared $\left(\delta N_{0}\right)^{2}$ in powers of $x$, where $x=e^{-\tilde{\beta}}$. Clearly, at very low temperatures $\left(\delta N_{0}\right)^{2}$ is independent of the number of particles $N$ (in both GCE and CE). Therefore, the exponential behaviour of the fluctuation of free bosons at low temperatures should remain valid even in the large $N$ limit. In the case of fermions the $\mathrm{CE}$ and GCE fluctuations are similar except at very low temperatures. Clearly, the CE and GCE curves of fermions approach zero differently as $T \rightarrow 0$. For high temperatures, on the other hand, GCE and CE give identical results, as expected.

It is to be noted from Fig. 2 (a) that the number fluctuation for free bosons and free fermions cross at a certain temperature, with the fermion one getting bigger at higher temperatures. This is to be expected, since the number of possibilities of creating holes within the fermi sea, and distributing particles above, increases more rapidly than for bosons, whose ground state has only one level. Semions $(g=1 / 2)$, which may be regarded either as interacting bosons, or as interacting fermions, have fluctuations intermediate between those of bosons $(g=0)$ and fermions $(g=1)$.

In one dimension, we have calculated the number fluctuation of bosons interacting via CSM using the ideal Haldane picture for both GCE and CE. The next challenge is to find the exact fluctuation using combinatorics. This has been done for free fermions which correspond to interacting bosons with interacting strength of $g=1$ [23]. Work is in progress to calculate this exact fluctuation for a general $g$.

\section{ACKNOWLEDGMENTS}

Two of us (RKB and MNT) thank the Institute of Mathematical Sciences, where part

of this work was done, for hospitality. This work was supported by Natural Sciences and Engineering Research Council (NSERC) of Canada. 


\section{APPENDIX: LOW TEMPERATURE EXPANSIONS IN GCE AND CE}

The low temperature behaviour of thermodynamic quantities in GCE are well known for bosonic systems. However, a comparison between GCE and CE calculations at low temperatures is not usually discussed for either Bose or Fermi systems. Further, making use of some asymptotic expansions, the CE fluctuation for bosons was earlier found to be linear right down to $T=0$. However, we give here the expansion of the fluctuation squared at low temperature in power of $x$, where $x=e^{\tilde{\beta}}$, and show that the CE fluctuation of bosons is in fact exponential at very low $T$. In GCE only expansion for fermions is possible, since the fluctuation tends to infinity at low temperature for bosons. Both expansions are possible in CE.

a. Grand Canonical Ensemble: In GCE the (fermionic) occupation number is:

$$
\left\langle n_{k}\right\rangle_{G C E}=\frac{1}{x^{\left(\mu-\epsilon_{k}\right)}+1}=\frac{1}{x^{(\mu-k-1 / 2)}+1}
$$

for a one dimensional system. The ground state number fluctuation squared is given by:

$$
\left\langle\left(\delta N_{0}\right)^{2}\right\rangle_{G C E}=\sum_{k=0}^{k_{F}} \frac{x^{(\mu-k-1 / 2)}}{\left[x^{(m u-k-1 / 2)}+1\right]^{2}}
$$

where $k_{F}$ is the Fermi level. At low temperatures, for the one-dimensional harmonic oscillator, $\mu \approx \mu_{0}=N$. Therefore,

$$
\left\langle\left(\delta N_{0}\right)^{2}\right\rangle_{G C E}=\sqrt{x}-2 x+4 x^{3 / 2}-4 x^{2}+6 x^{5 / 2}-8 x^{3}+8 x^{7 / 2}-8 x^{4} \ldots, \quad N \geq 4 .
$$

Note that the first few terms are independent of $N$.

b. Canonical Ensemble: In CE the first and second moments of the occupation number are known [24]:

$$
\begin{aligned}
& \left\langle n_{k}\right\rangle=\frac{1}{Z_{N}} \sum_{j}^{N}( \pm)^{j+1} x^{j \epsilon_{k}} Z_{N-j}, \\
& \left\langle n_{k}^{2}\right\rangle=\frac{1}{Z_{N}} \sum_{j}^{N}( \pm)^{j+1}[j \pm(j-1)] x^{j \epsilon_{k}} Z_{N-j},
\end{aligned}
$$

where the upper and lower signs refer to bosons and fermions respectively. Summing over the ground states up the Fermi level gives the fermionic ground state number:

$$
\left\langle N_{0}\right\rangle_{C E}=\frac{1}{Z_{N}} \sum_{j}^{N}(-1)^{j+1} x^{j / 2} \frac{1-x^{j N}}{1-x^{j}} Z_{N-j},
$$

where we let $\epsilon_{k}=k-1 / 2$, with $k=1,2, \ldots$ Therefore,

$$
\begin{aligned}
\left\langle\left(\delta N_{0}\right)^{2}\right\rangle_{C E} & =\sum_{k=1}^{N}\left\langle n_{k}\right\rangle-\sum_{k=1}^{N}\left\langle n_{k}\right\rangle^{2} \\
& =x+2 x^{4}+\ldots \quad, \quad N \geq 4
\end{aligned}
$$


where we have used $\left\langle n_{k}\right\rangle=\left\langle n_{k}^{2}\right\rangle$ and again the first few terms are independent of the system size $N$.

For bosons the ground state consists of one single lowest level, the low temperature expansion of the number fluctuation is given by

$$
\begin{aligned}
\left\langle\left(\delta N_{0}\right)^{2}\right\rangle_{C E} & =\left\langle n_{0}^{2}\right\rangle-\left\langle n_{0}\right\rangle^{2} \\
& =x+3 x^{2}+4 x^{3}+7 x^{4}+\ldots, \quad N>4 .
\end{aligned}
$$

Again as in the fermionic case the first few terms in the low temperature expansions are independent of the system size. Indeed it is interesting to note that in $\mathrm{CE}$, the fluctuations in both the systems approach zero as $T \rightarrow 0$ in exactly identical fashion. 


\section{REFERENCES}

[1] Landau L D and Lifshitz E M 1958 Statistical Physics (Addison-Wesley, Reading) p. 354

[2] Calogero F 1969 J. Math. Phys. 1021912197

[3] Sutherland B 1971 J. Math. Phys. 12 246; 1992 Phys. Rev. B 45907

[4] Haldane F D M 1991 Phys. Rev. Lett. 67937

Wu Yong Shi 1994 Phys. Rev. Lett. 73922

[5] Murthy M V N and Shankar R 1994 Phys. Rev, Lett. 73 3331; 1995 Phys. Rev, Lett. 75353

Ha Z N C 1994 Phys. Rev, Lett. 731574

[6] Bhaduri R K, Reimann S M, Viefers S, Ghose Choudhury A, and Srivastava M K 2000 J. Phys. B: At. Mol. Opt. Phys. 333895

[7] Van Zyl B P, Bhaduri R K, and Sigetich J 2002 J. Phys. B: At. Mol. Opt. Phys. 35 1251

[8] Fernandez J P and Mullin W J cond-mat/0203175

[9] Grossman S and Holthaus M 1996 Phys. Rev. E 543495 ; 1997 Phys. Rev. Lett. 79 3557

Politzer H D 1996 Phys. Rev. A $\mathbf{5 4} 5048$

Gajda M and Rzazewski K 1997 Phys. Rev. Lett. 782886

Wilkens M and Weiss C 1997 J. Mod. Opt. 441801

Navez P, Bitouk D, Gajda M, Idziaszek Z, and Rzazewski K 1997 Phys. Rev. Lett. 79 1879

[10] Illuminati F, Navez P and Wilkens M 1999 J. Phys. B 32 L461

[11] Viefers S, Hansson T H, and Leinaas J M 2001 J. Phys. B: At. Mol. Opt. Phys. 344609

[12] Mehta M L 1967 Random matrices and statistical theory of energy levels (Academic Press, New York) p. 199

[13] Sen D and Bhaduri R K 1999 Can. J. Phys. 77327

[14] Murthy M V N and Shankar R 1994 Phys. Rev, Lett. 723629

[15] Anderson P W 1992 Prog. Theor. Phys. Suppl. 10745

[16] Murthy M V N and Shankar R 1995 Physica B212 315

[17] Bernard D and Wu Yong Shi cond-mat/9404025 (unpublished)

[18] C. Nayak and F. Wilczek, Phys. Rev. Lett. 73, 2740 (1994).

[19] Isakov S B, Arovas D P, Myrheim J, and Polychronakos A P 1996 Phys. Lett. A 212 299

[20] Yang H S, Lee B, and Park C 1997 J. Korean Phys. Soc. 3014

[21] Aoyama T cond-mat/0005336 (unpublished)

[22] Bhaduri R K, Murthy M V N, and Srivastava M K 1996 Phys. Rev. Lett. 76165

[23] Tran M N, Murthy M V N, and Bhaduri R K 2001 Phys. Rev. E 63031105

[24] Parvan A S, Toneev V D, and Ploszajczak M, 2000 Nucl. Phys. A 676409

[25] Tran M N 2000 Ground State Number Fluctuations In Finite Bose And Fermi Systems M.Sc. Thesis

[26] Murthy M V N and Shankar R 1999 Phys. Rev. B 606517 


\section{FIGURES}

FIG. 1. The level flow of quasi-particle energy levels in CSM as a function of $g$.

FIG. 2. (a) The ground state fluctuation for the one-dimensional CSM system in GCE (Eq. (17)

and $\mathrm{CE}(E q$. (44) as a function of temperature for $N=10$. We show the results for fermions, bosons and also semions $(g=1 / 2)$.

FIG. 3. (b) Same as in (a) but using the low temperature expansions given in the Appendix 